\title{
Targeting the endocannabinoid system in the treatment of fragile $X$ syndrome
}

A. Busquets-Garcia ${ }^{1}$, M. Gomis-González ${ }^{1}$, T. Guegan ${ }^{1}$, C. Agustín-Pavón ${ }^{3, \S}$, A.

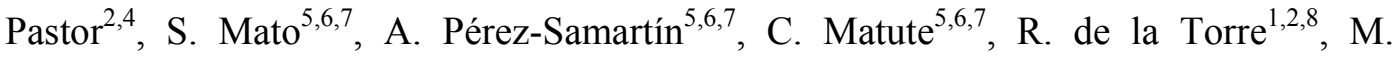
Dierssen $^{3,9}$, R. Maldonado ${ }^{1}$ and A. Ozaita ${ }^{1 *}$

${ }^{1}$ Departament de Ciències Experimentals i de la Salut. Universitat Pompeu Fabra, 08003 Barcelona, Spain.

${ }^{2}$ Grup de Recerca Clínica en Farmacologia Humana i Neurociències. IMIM-Hospital del Mar Medical Research Institute, 08003 Barcelona, Spain.

${ }^{3}$ Centre for Genomic Regulation (CRG), 08003 Barcelona, Spain.

${ }^{4}$ Facultat de Medicina, Universitat Autònoma de Barcelona, Barcelona, Spain.

${ }^{5}$ Laboratorio de Neurobiología, Departamento de Neurociencias, Universidad del País Vasco-UPV/EHU, 48940 Leioa, Spain.

${ }^{6}$ Achucarro Basque Center for Neuroscience-UPV/EHU, 48170 Zamudio, Spain.

${ }^{7}$ Instituto de Salud Carlos III (ISCIII; CIBERNED), 48940 Leioa, Spain.

${ }^{8}$ CIBER de Fisiopatología de la Obesidad y Nutrición (CB06/03), CIBEROBN, Hospital Clínico Universitario Santiago de Compostela, 15706 Santiago de Compostela, Spain.

${ }^{9}$ Centro de Investigación Biomédica en Red de Enfermedades Raras (CIBERER), 08003 Barcelona, Spain.

${ }^{\S}$ Current affiliation: EMBL/Systems Biology Unit, CRG, 08003 Barcelona, Spain

*Corresponding author:

Andrés Ozaita, Laboratori de Neurofarmacologia, Facultat de Ciències de la Salut i de la Vida, Universitat Pompeu Fabra, Parc de Recerca Biomèdica de Barcelona, C/ Doctor Aiguader 88, 08003 Barcelona, Spain. Phone: +34-93-3160823; Fax: + 34-93-3160901; e-mail: andres.ozaita@upf.edu

Running title: Targeting the endocannabinoid system in FXS.

Key words: Fragile X, memory, anxiety, nociception, audiogenic seizure, CB1, CB2, endocannabinoid system, mTOR. 


\section{Introductory paragraph}

Fragile X syndrome (FXS), the most common monogenic cause of inherited intellectual disability and autism ${ }^{1}$, is caused by the silencing of the FMR 1 gene leading to the loss of fragile X mental retardation protein (FMRP) ${ }^{2}$, a synaptically expressed RNA-binding protein regulating translation ${ }^{3}$. The $F m r 1$ knockout $(F m r 1 \mathrm{KO})$ model recapitulates the main traits of the disease ${ }^{4}$. An uncontrolled activity of metabotropic glutamate receptor 5 (mGluR5) $)^{5,6}$ and mammalian target of rapamycin (mTOR) signaling ${ }^{7,8,9}$ seem crucial in this pathology. The endocannabinoid system (ECS), is a key modulator of synaptic plasticity, cognitive performance, anxiety, nociception and seizure susceptibility ${ }^{10}$, all affected in FXS. Cannabinoid receptors, CB1 (CB1R) and CB2 (CB2R), are activated by phospholipid-derived endocannabinoids, and CB1R-driven long-term regulation of synaptic strength, frequently derived from mGluR5 activation ${ }^{11}$, is altered in several brain areas of Fmrl KO mice ${ }^{12,13,14}$. We found that CB1R blockade in Fmrl ${ }^{-/ y}$ mice, through pharmacological and genetic approaches, normalized the cognitive impairment, nociceptive desensitization, susceptibility to audiogenic seizures, over-activated mTOR signaling, and altered spine morphology, while pharmacological blockade of CB2R normalized anxiolytic-like behavior. Some of these traits were also reversed by pharmacological inhibition of mTOR or mGluR5. Thus, blockade of ECS is a potential therapeutic approach to normalize specific alterations in FXS. 


\section{Main text}

FXS, the principal monogenic syndrome leading to inherited intellectual disability and autism $^{15}$, is caused by an unstable expansion of CGG repeats in the 5'UTR of the gene FMR1, producing the loss in the expression of FMRP ${ }^{2}$. Among others, an uncontrolled activity of group I metabotropic glutamate receptors, mainly mGluR5 $5^{5,6}$, reduced GABAergic transmission $^{16,17}$, and an enhanced mTOR signaling ${ }^{7}$ seem to play a causal role in FXS deficits.

Synaptic activation of mGluR5 promotes the synthesis of endocannabinoids ${ }^{11}$, triggering CB1R-mediated long-term depression (LTD) of excitatory and inhibitory transmission ${ }^{10}$. Noteworthy, deregulated mGluR5 driven LTD has been recently described in several brain areas of adult $F m r l^{-/ y}$ mice $^{12,14}$. At the molecular level, ECS activation enhances the phosphatidylinositol-3-kinase (PI3K)/protein kinase $\mathrm{B}$ $(\mathrm{Akt}) / \mathrm{mTOR} / \mathrm{p} 70 \mathrm{~S} 6$ kinase $(\mathrm{p} 70 \mathrm{~S} 6 \mathrm{~K})$ signaling pathway in the hippocampus ${ }^{18,19}$. This transduction pathway is closely related to synaptic plasticity ${ }^{20}$, is de-regulated in $\mathrm{Fmrl}^{-}$ /y mice $^{7}$, and mediates particular behavioral effects of cannabinoids ${ }^{21}$, but its pharmacological inhibition has never been tested in the Fmrl KO mice. Since the ECS has putative regulatory capacity on most FXS traits such as cognition, anxiety-like behavior, antinociception and neuronal plasticity ${ }^{10}$, we sought to characterize the ECSmTOR pathway as a potential target for therapeutic intervention in FXS.

We found that acute administration of the CB1R antagonist rimonabant in $\mathrm{Fmrl}^{-/ \mathrm{y}}$ mice during the consolidation phase of the object-recognition memory test ameliorated their cognitive deficit, without affecting the performance of wild-type (WT) littermates (Fig. 1a). 
Under similar conditions, acute blockade of CB2R with AM630, or mGluR5 with MTEP were not effective (Fig. 1b,c). Interestingly, acute temsirolimus administration also prevented this memory impairment (Fig. 1d).

When chronically administered, rimonabant, MTEP and temsirolimus, but not AM630, were equally effective at preventing the cognitive deficit in $F m r l^{-/ y}$ mice (Fig. 1, e-h). Notably, acute intra-hippocampal microinjection of rimonabant during objectrecognition memory consolidation improved the cognitive deficit in $F m r l^{-/ y}$ mice (Fig. $1 \mathrm{i}$ and Supplementary Fig. 2) pointing to the crucial role of CB1R activity in this brain region for the memory deficit. We next tested whether mGluR5 and CB1R inhibition converge on normalizing the cognitive deficit. MTEP has a short half-life ${ }^{6}$ and was only effective after chronic administration. Therefore, mice were chronically treated with a sub-optimal dose of MTEP $\left(5 \mathrm{mg} \mathrm{kg}^{-1}\right)$ receiving an acute administration of rimonabant after the training session. We observed an enhanced performance of the combined treatment compared with both treatments separately (Fig. 1j), pointing to a complementary involvement of both mechanisms.

Recent studies propose that Fmrp loss affects the efficacy of mGluR5-driven endocannabinoid production machinery in different brain areas ${ }^{12,13,14}$. Under our experimental conditions, we did not detect differences between WT and $F m r l^{-/ y}$ mice in brain basal levels of the two main endocannabinoids, 2-arachidonoylglycerol (WT vs. $F m r 1^{-/ y}: 100.0 \pm 5.7 \%$ vs. $109.7 \pm 4.8 \%, \mathrm{P}=0.213$ ) and anandamide (WT vs. $F m r l^{-/ y}$ : $100.0 \pm 4.9 \%$ vs. $107.4 \pm 3.0 \%, \mathrm{P}=0.223$ ), nor in the hippocampal expression of several components of the ECS (Supplementary Fig. 3) in agreement with previous studies $^{12,13}$. We next examined CB1R-dependent modulation of GABAergic transmission in the hippocampus CA1 area. To estimate possible differences in synaptic endocannabinoid tone, we analyzed the effect of pharmacological CB1R blockade on 
baseline inhibitory postsynaptic currents (IPSCs) in hippocampal slices from both genotypes $^{22}$. Bath application of rimonabant increased IPSC amplitude to a similar extent in WT and $F m r 1^{-/ y}$ mice (Supplementary Fig. 4a), suggesting that Fmrp loss does not affect the level of tonic CB1R activation at GABAergic synapses. Moreover, inhibitory long-term depression LTD (i-LTD) initiated by mGluR $1 / 5^{10}$, a form of synaptic plasticity mediated by endocannabinoids, was similarly induced by high frequency stimulation (HFS) and by theta burst stimulation (TBS) in both WT and $F m r 1^{-/ y}$ mice (Fig. 2a,b). Consistently, we detected no differences between genotypes in the magnitude of $i-L T D$ elicited by bath application of the mGluR1/5 agonist DHPG, or in the ability of the CB1R agonist CP55,940 to inhibit GABAergic transmission onto CA1 neurons (Supplementary Fig. 4). All these data suggest that Fmrp removal does not significantly affect the ECS modulation of inhibitory transmission and long-term synaptic plasticity in hippocampal CA1 area.

The hippocampus of $\mathrm{Fmrl}^{-/ \mathrm{y}}$ mice exhibited a marked increase in the phosphorylation of p70S6K(T389) (Fig. 2c) that localized to CA1 pyramidal neurons, but not GABAergic interneurons (Fig. 2d). Remarkably, the phosphorylation status of p70S6K(T389) and Akt(S473), both involved in mTOR pathway signaling, was specifically enhanced in the hippocampus of $F m r 1^{-/ y}$ mice (Fig. 2e and Supplementary Fig. 5). Interestingly, both rimonabant and MTEP chronic treatment normalized the phosphorylation levels of $\mathrm{Akt}(\mathrm{S} 473)$ and p70S6K(T389) (Fig. 2e) in hippocampal tissue. Moreover, the enhanced dendritic spine density of CA1 pyramidal neurons in $F_{m r l^{-/ y}}$ mice was also normalized by rimonabant chronic treatment (Fig. 2f). When spines were classified based on their morphology, rimonabant-treated $F m r I^{-/ y}$ mice showed a decrease in thin/stubby (immature) spines and an increase in mushroom/wide 
(mature) spines compared to vehicle-treated $F m r l^{-/ y}$ mice (Fig. 2g), similarly to other normalizing interventions ${ }^{6,9}$.

Chronic MTEP and rimonabant normalized in adult $F m r 1^{-/ y}$ mice both the cognitive deficit and the hippocampal mTOR signaling pathway, and showed an additive effect when both treatments were combined, revealing the significance of ECS tone and mTOR activity in this cognitive deficit. mTOR signaling is crucial in memory consolidation $^{23}$ and genetic regulation of this signaling pathway in $\mathrm{Fmrl}^{-/ \mathrm{y}}$ mice has prevented some of the $F m r l^{-/ y}$ features ${ }^{9}$. We previously showed that mTOR overactivation in CA1 pyramidal neurons is directly involved in the cognitive deficit induced by ECS activation ${ }^{18,19,21}$, which can explain the efficacy of rimonabantmediated CB1R blockade to reverse the cognitive impairment in $F m r l^{-/ y}$ mice. The fact that temsirolimus also prevented the object-recognition memory deficit in $\mathrm{Fmrl}^{-\mathrm{y}}$ mice reinforces the concept that mTOR signaling over-activation plays a key role in this deficiency ${ }^{24}$ and demonstrates the possibility of using these pharmacological approaches as a potential therapy. Given that CA1 hippocampal GABAergic presynaptic membranes display 10-20 fold heavier expression of CB1R than glutamatergic ones ${ }^{25}$, rimonabant might help normalizing the balance between excitatory and inhibitory inputs, already altered in FXS, and lead to an improved output in the cognitive test, by facilitating inhibitory transmission onto CA1 pyramidal cells ${ }^{26}$ (Supplementary Fig. 4). The balancing effect of rimonabant may also fit with the effect of other therapeutic approaches aiming to reestablish the excitatory/inhibitory balance, such as the mGluR5 antagonist $\mathrm{CTEP}^{6}$, or the NMDA receptor antagonist memantine ${ }^{27}$, both reducing the excitatory drive, and the $\mathrm{GABA}_{\mathrm{B}}$ receptor agonist arbaclofen ${ }^{17}$, increasing the inhibitory drive. 
Adult $\mathrm{Fmrl}^{-\mathrm{y}}$ mice show a reduced-anxiety phenotype in the elevated plus-maze (Fig. 3, a-d). This trait was insensitive to rimonabant or temsirolimus (Fig. 3a,d). Instead, acute CB2R and mGluR5 blockade (Fig. 3b,c) normalized such a phenotype, without modifying the anxiety-like behavior in WT mice (Fig. 3b,c). In this regard, CB2R have been involved in the anxiolytic-like effects produced by an enhanced level of 2arachidonoylglycerol $^{18}$, while CB1R were associated to the anxiolytic-like effect of anandamide accumulation ${ }^{18}$. Since we used doses of rimonabant and AM630 that did not affect the anxiety-like behavior in WT mice, we conclude that the reduced anxiety phenotype in $\mathrm{Fmrl}^{-/ \mathrm{y}}$ mice may specifically involve CB2R signaling.

Another characteristic of adult $F m r 1^{-/ y}$ mice, such as the diminished responses to inflammatory pain, which is sensitive to mGluR5 blockade ${ }^{28}$, was studied using the formalin test. This phenotype could be relevant to the self-injurious behavior that often appears in FXS, and was only normalized by rimonabant (Fig. 3e), similar to previously reported in a different model of peripheral and central nociceptive sensitization ${ }^{29}$.

In addition, the marked sensitivity to audiogenic seizures in $F m r l^{-/ y}$ mice $^{30}$ was bluntly decreased by the pre-treatment with rimonabant, AM630 and temsirolimus (Fig. 3f), similarly to that previously reported through mGluR5 antagonism ${ }^{6}$. The involvement of CB2R and mTOR signaling in this phenotypic characteristic reveals a new central effect of these targets.

To further support the contribution of CB1R signaling in behavioral and biochemical manifestations of FXS, we generated a double mutant Fmrl KO line with a reduced expression of CB1R $\left(\mathrm{Cnrl}^{+/-}\right)$. We found that those rimonabant-sensitive features in $F_{m r 1^{-/ y}}$ mice, such as the cognitive deficit (Fig. 4a), the nociceptive desensitization (Fig. 4b), the audiogenic seizure susceptibility (Fig. 4c), and the over-activation of the 
mTOR signaling pathway (Fig. 4d), were bluntly prevented by the genetic decrease of CB1R expression.

Taken together our results reveal the involvement of the ECS in specific behavioral, synaptic and molecular manifestations of FXS. We demonstrate that the pharmacological or genetic blockade of CB1R reestablish some of FXS traits such as the cognitive impairment, the decreased nociceptive response, the increased susceptibility to audiogenic seizures, and the normalization of the mTOR pathway in the hippocampus. Moreover, CB2R play an important role in the regulation of the anxiolytic-like behavior and the increased susceptibility to audiogenic seizures. In conclusion, our data point to the regulation of the ECS and mTOR pathway as potential targets for the development of new therapeutic approaches in FXS (Supplementary Fig. 8). 


\section{Bibliography}

1. De Vries BB, Halley DJ, Oostra BA. \& Niermeijer MF. The fragile X syndrome. $J$. Med. Genet. 35, 579-589 (1998).

2. Verkerk AJ. et al. Identification of a gene (FMR-1) containing a CGG repeat coincident with a breakpoint cluster region exhibiting length variation in fragile $\mathrm{X}$ syndrome. Cell 31, 905-914 (1991).

3. Darnell JC. et al. FMRP stalls ribosomal translocation on mRNAs linked to synaptic function and autism. Cell 22, 247-261 (2011).

4. Bakker CE. et al. Fmr1 knockout mice: a model to study fragile X mental retardation. The Dutch-Belgian Fragile X Consortium. Cell 78, 23-33 (1994).

5. Bear MF, Huber KM. \& Warren ST. The mGluR theory of fragile X mental retardation. Trends Neurosci. 27, 370-377 (2004).

6. Michalon A. et al. Chronic pharmacological mGlu5 inhibition corrects fragile $\mathrm{X}$ in adult mice. Neuron 74, 49-56 (2012).

7. Sharma A. et al. Dysregulation of mTOR signaling in fragile X syndrome. $J$. Neurosci. 30, 694-702 (2010).

8. Hoeffer CA. et al. Altered mTOR signaling and enhanced CYFIP2 expression levels in subjects with fragile X syndrome. Genes Brain Behav. 11, 332-341 (2012).

9. Bhattacharya A. et al. Genetic removal of p70 S6 kinase 1 corrects molecular, synaptic, and behavioral phenotypes in fragile X syndrome mice. Neuron 76, 325337 (2012).

10. Kano M. et al. Endocannabinoid-mediated control of synaptic transmission. Physiol. Rev. 89, 309-380 (2009).

11. Varma N, Carlson GC, Ledent C. \& Alger BE. Metabotropic glutamate receptors drive the endocannabinoid system in hippocampus. J. Neurosci. 21, RC188 (2001).

12. Zhang L. \& Alger BE. Enhanced endocannabinoid signaling elevates neuronal excitability in fragile X syndrome. J. Neurosci. 30, 5724-5729 (2010)

13. Maccarrone M. et al. Abnormal mGlu 5 receptor/endocannabinoid coupling in mice lacking FMRP and BC1 RNA. Neuropsychopharmacology. 35, 1500-1509 (2010).

14. Jung KM. et al. Uncoupling of the endocannabinoid signalling complex in a mouse model of fragile X syndrome. Nat. Commun. 3, 1080 (2012).

15. Penagarikano O., Mulle JG. \& Warren ST. The pathophysiology of fragile $x$ syndrome. Annu. Rev. Genomics Hum. Genet. 8, 109-129 (2007).

16. D'Hulst C. \& Kooy RF. The GABAA receptor: a novel target for treatment of fragile X? Trends Neurosci. 30, 425-431 (2007).

17. Henderson C. et al. Reversal of disease-related pathologies in the fragile $\mathrm{X}$ mouse model by selective activation of GABA(B) receptors with arbaclofen. Sci. Transl. Med. 4, 152ra128 (2012).

18. Busquets-Garcia A. et al. Differential role of anandamide and 2arachidonoylglycerol in memory and anxiety-like responses. Biol. Psychiatry. 70, 479-486 (2011).

19. Puighermanal E., Busquets-Garcia A., Maldonado R. \& Ozaita A. Cellular and intracellular mechanisms involved in the cognitive impairment of cannabinoids. Philos. Trans. R. Soc. Lond. B Biol. Sci. 367, 3254-3263 (2012).

20. Hoeffer CA. \& Klann E. mTOR signaling: at the crossroads of plasticity, memory and disease. Trends Neurosci. 33, 67-75 (2010).

21. Puighermanal E. et al. Cannabinoid modulation of hippocampal long-term memory is mediated by mTOR signaling. Nat Neurosci. 12, 1152-1158 (2009). 
22. Lafourcade M. et al. Nutritional omega-3 deficiency abolishes endocannabinoidmediated neuronal functions. Nat Neurosci. 14, 345-350 (2011).

23. Richter JD. \& Klann E. Making synaptic plasticity and memory last: mechanisms of translational regulation. Genes Dev. 23, 1-11 (2009).

24. Troca-Marín JA, Alves-Sampaio A. \& Montesinos ML. Deregulated mTORmediated translation in intellectual disability. Prog. Neurobiol. 96, 268-282 (2012).

25. Kawamura Y. et al. The CB1 cannabinoid receptor is the major cannabinoid receptor at excitatory presynaptic sites in the hippocampus and cerebellum. $J$. Neurosci. 26, 2991-3001 (2006).

26. Kang-Park MH. et al. Differential sensitivity of GABA A receptor-mediated IPSCs to cannabinoids in hippocampal slices from adolescent and adult rats. $J$. Neurophysiol. 98, 1223-1230 (2007).

27. Wei H. et al. The therapeutic effect of memantine through the stimulation of synapse formation and dendritic spine maturation in autism and fragile $\mathrm{X}$ syndrome. PLoS One. 7, e36981 (2012).

28. Price TJ. et al. Decreased nociceptive sensitization in mice lacking the fragile $X$ mental retardation protein: role of mGluR1/5 and mTOR. J. Neurosci. 27, 13958-13967 (2007).

29. Guindon J. \& Hohmann AG. The endocannabinoid system and pain. CNS Neurol. Disord. Drug Targets. 8, 403-421 (2009).

30. Chen L. \& Toth M. Fragile X mice develop sensory hyperreactivity to auditory stimuli. Neuroscience. 103, 1043-1050 (2001). 


\section{Material and Methods}

Animals: Fmr1 knockout (KO) mice in FVB background (Fmr1 KO, FVB.129P2$P d e 6 b^{+}$Tyr $^{c-c h} F m r 1^{\text {tmlCgr }} / \mathrm{J}$ ) and wild-type mice (WT, FVB.129P2-Pde6b ${ }^{+}$Tyr $^{c-c h} / \mathrm{AntJ}$ ) were purchased from The Jackson Laboratory and crossed to obtain $F m r 1^{-/ y}$ and WT littermates. Fmrl KO mice in C57BL/6J congenic background (B6.129P2$\left.F m r 1^{t m l C g r} / \mathrm{J}\right)^{4}$ were obtained from the Baylor College of Medicine Mouse Facility. Double mutant mice $\left(\mathrm{Fmrl}^{-/ \mathrm{y}} / \mathrm{Cnrl}^{+/-}\right)$mice in $\mathrm{C} 57 \mathrm{BL} / 6 \mathrm{~J}$ background were initially generated by crossing homozygous female mice carrying the $F m r l$ mutation $\left(\mathrm{Fmrl}^{-/-}\right)$ with homozygous male mice carrying the $\mathrm{Cnrl}$ mutation $\left(\mathrm{Cnrl}^{-/}\right)$. Subsequently, animals used for experimentation were derived from the crossing of female $\mathrm{Fmrl}^{+/-}$ $/ \mathrm{Cnrl}^{+/+}$with male $\mathrm{Fmrl}^{+/ \mathrm{y}} / \mathrm{Cnrl}^{+/-}$. All experimental animals were bred in-house at the Barcelona Biomedical Research Park (PRBB) Animal Facility. Fmr $1^{-/ y}$ and WT mice were used at 12 to 16 weeks of age, except to study audiogenic seizure susceptibility (21-23 days old). Mice were housed four per cage in a temperature $\left(21 \pm 1^{\circ} \mathrm{C}\right)$ and humidity (55 $\pm 10 \%)$ controlled environment. Food and water were available ad libitum. All the experiments were performed during the light phase of a $12 \mathrm{~h}$ light/dark cycle (lights on at 8 am and off at $8 \mathrm{pm}$ ). Animals were handled for one week before starting the experiments. All animal procedures followed the standard ethical guidelines (European Communities Directive 86/60-EEC) and were approved by the local ethical committee (Comitè Ètic d'Experimentació Animal-Parc de Recerca Biomèdica de Barcelona, CEEA-PRBB). The PRBB has also the Animal Welfare Assurance (\#A5388-01, IACUC Approval Date 06/08/2009) granted by the Office of Laboratory Animal Welfare (OLAW) of the National Institutes of Health (USA). All behavioral tests were performed by researchers blind to the different experimental groups. 
Drugs and treatments: Rimonabant was obtained from Sanofi-Aventis (SanofiAventis Recherche); 6-Iodo-2-methyl-1-[2-(4-morpholinyl)ethyl]-1H-indol-3-yl](4methoxyphenyl)methanone (AM630), DL-2-Amino-5-phosphonopentanoic acid (DAPV), 2,3-Dioxo-6-nitro-1,2,3,4-tetrahydrobenzo[f]quinoxaline-7-sulfonamide disodium salt (NBQX) and (S)-3,5-Dihydroxyphenylglycine (DHPG) from Tocris Bioscience; 3-((2-Methyl-4-thiazolyl)ethynyl)pyridine (MTEP) was a kind gift from Merck Research Laboratories; temsirolimus (CCI-779) from LC Laboratories. CP55,940 and picrotoxin were purchased from Sigma-Aldrich. Rimonabant, AM630 and MTEP were diluted in 5\% ethanol: 5\% cremophor-EL: 90\% saline. Temsirolimus was dissolved in 2\% ethanol: $8 \%$ cremophor-EL: $90 \%$ saline $^{31}$. All compounds injected intraperitoneally (i.p.) were administered in a volume of $10 \mathrm{ml}$ per $\mathrm{kg}$.

Intra-hippocampus administration of rimonabant: Rimonabant $(1.5 \mu \mathrm{g}$ per $0.5 \mu \mathrm{l})$ was directly administered into the hippocampus as described elsewhere ${ }^{32}$. After recovery (8 days), mice were administered bilaterally with rimonabant or its vehicle after the training session in the object-recognition test. After completion of the experimental sequence, cannula position was verified histologically (Supplementary Fig. 2) as described previously ${ }^{32}$.

Endocannabinoid quantification: Endocannabinoids, 2-arachidonoylglycerol (2-AG) and anandamide (AEA), were analyzed as described previously ${ }^{18}$ using half right or left brain (including forebrain, midbrain and hindbrain) (220-250 mg approximately). The endogenous concentrations of 2-AG and AEA were calculated based on the response of the deuterated analogues and expressed as percentage of control WT mice. 
Immunoblot analysis: Tissues from different brain regions (hippocampus, frontal cortex, striatum, amygdala, and cerebellum) from WT and $F m r l^{-/ y}$ mice were analyzed in basal conditions. Hippocampal tissues from WT and $F m r l^{-/ y}$ mice after chronic pharmacological treatment or from the double mutant mouse line $\left(\mathrm{Fmrl}^{+/ \mathrm{y}} / \mathrm{Cnrl}^{+/+}\right.$, $\mathrm{Fmrl}^{-/ \mathrm{y}} / \mathrm{Cnrl}^{+/+}$and $\mathrm{Fmrl}^{-/ \mathrm{y}} / \mathrm{Cnrl}^{+/-}$) were also analyzed. Tissues were processed as previously described ${ }^{21}$. The antibodies used for immunoblot detected: Fmrp (cat. \# ab17722, 1:500), monoacylglycerol lipase (MAGL) (cat. \# ab24701, 1:1,000), fatty acid amide hydrolase (FAAH) (cat. \# ab54615, 1:500), (Abcam); phospho-p70S6K(T389) (cat. \# 9234, 1:800), p70S6K (cat. \# 9202, 1:500), phospho-Akt(S473) (cat. \# 4051, 1:200), Akt (cat. \# 9272, 1:2000) (Cell Signaling Technology); diacylglycerol lipase alpha (DGL-alpha) (cat. \# GP-Af380-1, 1:400), N-acyl phosphatidylethanolamine phospholipase D (NAPE-PLD) (cat. \# GP-Af720-1 , 1:1,000), mGluR5 (cat. \# GPAf270-1, 1:1,000), CB1 receptor (cat. \# Rb-Af380-1, 1:1,000) (Frontier Science); glyceraldehyde-3-phosphate dehydrogenase (GAPDH) (cat. \# sc-32233, 1:5,000) (Santa Cruz Biotechnology). Optical densities of relevant immunoreactive bands were quantified after acquisition on a ChemiDoc XRS System (Bio-Rad) controlled by The Quantity One software v 4.6.3 (Bio-Rad).

Immunofluorescence: Tissue was prepared as described previously ${ }^{21}$ Hippocampi containing slices were rinsed in $0.1 \mathrm{M}$ phosphate buffer (PB), incubated in permeabilizing/blocking solution $(0.2 \%$ Triton $\mathrm{X}-100,5 \%$ bovine serum albumin in $\mathrm{PB})$ for $2 \mathrm{~h}$, and overnight at $4{ }^{\circ} \mathrm{C}$ with antibody to phospho-p70S6K(T389) (cat. \# 9206 , 1:100, mouse) and antibody to GAD65/67 (cat. \# AB1511, 1:100, rabbit, Millipore) antibodies. Next day, after rinsing in PB, sections were incubated with the antibody to mouse-Alexa Fluor 647 (cat. \# 115-165-146, 1:500, Invitrogen) or the antibody to 
rabbit-Cy3 (cat. \# 111-225-144, 1:500, Jackson ImmunoResearch Laboratories). After rising, slices were mounted onto gelatin-coated slides with Mowiol mounting media. Confocal images were obtained as previously described ${ }^{21}$.

Slice preparation and electrophysiology: $F m r 1^{-/ y}$ and WT mice were anesthetized with isoflurane and their brains were removed to a chilled sucrose based solution (in mM: 215 sucrose, $2.5 \mathrm{KCl}, 26 \mathrm{NaHCO}_{3}, 1.6 \mathrm{NaH}_{2} \mathrm{PO}_{4}, 1 \mathrm{CaCl}_{2}, 4 \mathrm{MgCl}_{2}, 4 \mathrm{MgSO}_{4}, 20$ glucose, $1.3 \mathrm{mM}$ ascorbic acid), and coronal brain slices (350 $\mu \mathrm{m}$ thick) were cut with a Vibratome Series 3000 Plus-Tissue Sectioning System (Ted Pella, Inc). Sections containing the hippocampus were stored for $30 \mathrm{~min}$ at $30-32{ }^{\circ} \mathrm{C}$ in recovery solution (in mM: $62 \mathrm{NaCl}, 2.5 \mathrm{KCl}, 25 \mathrm{NaHCO}_{3}, 1.4 \mathrm{NaH}_{2} \mathrm{PO}_{4}, 1.1 \mathrm{CaCl}_{2}, 3.3 \mathrm{MgCl}_{2}, 2 \mathrm{MgSO}_{4}, 15$ glucose, 108 sucrose), and then moved into room temperature low-calcium artificial cerebrospinal fluid (ACSF) (in mM: $124 \mathrm{NaCl}, 2.5 \mathrm{KCl}, 25 \mathrm{NaHCO}_{3}, 1.2 \mathrm{NaH}_{2} \mathrm{PO}_{4}$, 1.25 $\mathrm{CaCl}_{2}, 2.6 \mathrm{MgCl}_{2}, 10$ glucose) for at least $1 \mathrm{~h}$. Experiments were conducted at room temperature in a submersion-type recording chamber perfused at $1.5 \mathrm{ml} / \mathrm{min}$ with ACSF (same as low-calcium ACSF, with the following exception: $2.5 \mathrm{mM} \mathrm{CaCl}_{2}$ and $1.3 \mathrm{mM}$ $\mathrm{MgCl}_{2}$ ). All solutions were saturated with $95 \% \mathrm{O}_{2}$ and $5 \% \mathrm{CO}_{2}, \mathrm{pH} 7.4$.

Whole cell recordings were made on visualized CA1 pyramidal neurons voltage clamped at $-70 \mathrm{mV}$ with a pipette $\left(2-4 \mathrm{M} \Omega\right.$ ) containing (in $\mathrm{mM}$ ): $90 \mathrm{CsCH}_{3} \mathrm{SO}_{3}, 50$ CsCl, 10 HEPES, $1 \mathrm{MgCl}_{2}$, 0.2 EGTA, $4 \mathrm{Mg}^{2+}$-ATP, 0.3 Tris-GTP, 5 QX314, pH 7.25, $285 \mathrm{mOsm}$ ). Inhibitory postsynaptic currents (IPSCs) were isolated by adding the NMDA and AMPA/KA receptor antagonists (25 $\mu \mathrm{M}$ D-AP5 and $10 \mu \mathrm{M}$ NBQX) to the ACSF. To evoke monosynaptic currents, $150 \mu \mathrm{sec}$ duration stimuli were delivered every $30 \mathrm{sec}$ through a patch pipette filled with ACSF and placed in stratum radiatum. Series resistance (typically 10-20 M 2 ) was monitored through the experiments by applying a - 
$2 \mathrm{mV}$ hyperpolarizing pulse before each evoked IPSC, and cells with more than $20 \%$ change in this parameter were excluded from the analysis. LTD was induced by high frequency stimulation (HFS, 2 trains of 100 pulses at $100 \mathrm{~Hz}, 20 \mathrm{sec}$ apart), or by theta burst stimulation (TBS, 10 bursts of 5 stimuli applied at $100 \mathrm{~Hz}$ with $200 \mathrm{msec}$ interburst intervals repeated 4 times, $5 \mathrm{sec}$ apart). The magnitude of LTD and the effect of the different drugs were estimated by comparing averaged responses corresponding to the last $5 \mathrm{~min}$ of the experiment with baseline averaged responses $5 \mathrm{~min}$ before the induction protocol or the beginning of drug application. Recordings were performed with a MultiClamp 700B (Axon Instruments), and output signals were filtered at $3 \mathrm{KHz}$. Data were digitized $(10 \mathrm{KHz})$ on a DigiData 1332A (Axon Instruments). Data were collected using Clampex 9.2 and IPSC amplitude was analyzed using Clampfit 9.2.

Dendritic spine morphology analysis: Dendritic spine analysis was performed as previously described ${ }^{33}$ in mice that received a chronic administration of rimonabant (1 $\left.\mathrm{mg} \mathrm{kg}^{-1}, 7 \mathrm{~d}\right)$ or its vehicle. Brains were extracted after perfusion (4\% PFA in PB) $3 \mathrm{~h}$ after the last administration of rimonabant or vehicle solution on the seventh day of treatment. Secondary to tertiary dendrites of pyramidal neurons from the CA1 region of the hippocampus were chosen for spine analysis based on the criteria described previously $^{33}$.

Object-recognition task: Object-recognition memory was assayed as described previously $^{21}$. All acute treatments and the last administration of chronic treatments were performed after the training session in the object-recognition test. 
Anxiety-like response: Anxiety-like responses were evaluated as previously described $^{18}$. Briefly, 5 min test sessions were performed $120 \mathrm{~min}$ after drug administration. The time spent in the open arms as well as the total number of entries in the arms was recorded.

Nociceptive response: The late phase nociceptive response (corresponding to inflammatory pain $)^{34}$ was recorded during $20 \mathrm{~min}$, starting $20 \mathrm{~min}$ after formalin $(5 \%$, $20 \mu \mathrm{L}$ ) injection into de right hind paw. All drugs were injected 20 minutes before the formalin injection. The nociceptive behavior (licking) was measured.

Audiogenic seizure sensitivity: Mice were placed individually into an observation chamber, a glass cylinder (40 $\mathrm{cm}$ high, $16 \mathrm{~cm}$ diameter), allowing the exploration for 1 min. Next, a bell-ring (100 dB) rung for $30 \mathrm{sec}$. Animals were tested only once. Seizure activity was scored as follows: no response, 0 ; wild running, 1; clonic seizure, 2; tonic seizure, 3; status epilepticus/respiratory arrest/death, 4. Drugs were administered 30 minutes before the test.

Statistical analysis: Results are reported as mean \pm s.e.m. Most of the experiments were evaluated by one-way analysis of variance (ANOVA) followed by the Dunnett's post-hoc test when required. Two-way ANOVA (rimonabant, AM630, MTEP, temsirolimus treatment vs. WT or $F m r l^{-/ y}$ genotype) was used when required. Statistical comparisons of electrophysiological data were performed using Student's t test. Comparisons were considered statistically significant when $P<0.05$. 


\section{References for online Material and Methods section}

4. Bakker CE. et al. Fmr1 knockout mice: a model to study fragile X mental retardation. The Dutch-Belgian Fragile X Consortium. Cell 78, 23-33 (1994).

18. Busquets-Garcia A. et al. Differential role of anandamide and 2arachidonoylglycerol in memory and anxiety-like responses. Biol. Psychiatry. 70, 479-486 (2011).

21. Puighermanal E. et al. Cannabinoid modulation of hippocampal long-term memory is mediated by mTOR signaling. Nat Neurosci. 12, 1152-1158 (2009).

31. Puighermanal E, Busquets-Garcia A, Gomis-González M, Marsicano G, Maldonado R, Ozaita A. Dissociation of the pharmacological effects of THC by mTOR blockade. Neuropsychopharmacology. Jan 28 (2013).

32. Castañé A., Maldonado R. \& Valverde O. Role of different brain structures in the behavioural expression of WIN 55,212-2 withdrawal in mice. Br J Pharmacol. 142, 1309-1317 (2004).

33. Guegan T.et al. Operant behavior to obtain palatable food modifies neuronal plasticity in the brain reward circuit. Eur. Neuropsychopharmacol. May 19 (2012).

34. Noble F. et al. Pain-suppressive effects on various nociceptive stimuli (thermal, chemical, electrical and inflammatory) of the first orally active enkephalinmetabolizing enzyme inhibitor RB 120. Pain. 73, 383-391 (1997). 


\section{Figure legends}

Figure 1. Pharmacological modulation of object-recognition memory impairment in the $\mathrm{Fmrl}^{-/ \mathrm{y}}$ mice. Cognitive effect of the acute treatment with rimonabant (a), AM630 (b), MTEP (c) and temsirolimus (d) or their vehicles in $F m r 1^{-/ y}$ and WT mice ( $n=6-8$ per group). All drugs were administered after the training phase and discrimination indexes were obtained $24 \mathrm{~h}$ after training. Effect of chronic treatment with rimonabant (e), AM630 (f), MTEP (g) and temsirolimus (h) or their vehicles in the object-recognition performance of $\mathrm{Fmrl}^{-/ \mathrm{y}}$ and WT mice ( $n=6-8$ per group). All drugs were administered once daily for 7 days $(\mathbf{e}-\mathbf{h})$. The last drug administration was performed after the training session in the object-recognition assay and cognitive performance was measured $24 \mathrm{~h}$ later. (i) Cognitive performance after acute bilateral intrahippocampal microinjection of rimonabant $(1.5 \mu \mathrm{g}$ in $0.5 \mu \mathrm{l}$ per side) or vehicle after the training session. The discrimination index values were obtained $24 \mathrm{~h}$ after training and intra-hippocampal drug delivery. (j) The repeated administration of a suboptimal dose of MTEP combined with an acute administration of rimonabant improves the cognitive performance in obtained by any of both treatments separately. None of the treatments affected overall exploration (Supplementary Fig. 1). Data are expressed as mean \pm s.e.m. $* * * P<0.001, * * P<0.01\left(F m r l^{-/ y}\right.$ versus $\left.\mathrm{WT}\right), \# P<0.05, \# \# P<0.01$, $\# \# \#>0.001$ (rimonabant/temsirolimus versus vehicle), $\& P<0.05$ (MTEP chronic versus MTEP chronic + acute rimonabant).

Figure 2. Cellular and molecular effects of pharmacological treatment in the adult $\mathrm{Fmr1}^{-/ \mathbf{y}}$ mice. (a, b) Long-term depression of inhibitory synaptic transmission (i-LTD) is not altered in the hippocampus of $F m r l^{-/ y}$ mice. Sample traces of IPSCs $(\mathbf{a}, \mathbf{b})$ and 
average time courses of i-LTD induced by high frequency stimulation (HFS) (a) and theta burst stimulation (TBS) (b) in CA1 pyramidal cells of WT and $F m r 1^{-/ y}$ mice. Traces obtained at time points 1 and 2 are superimposed. Scale bar: 100 pA, 50 msec. (c) Immunofluorescence detection of p-p70S6K(T389) in the CA1 hippocampal region of $\mathrm{Fmrl}^{-/ \mathrm{y}}$ and WT mice. Note the enhanced staining in the stratum pyramidale (Pyr) and stratum radiatum (s.r.) but not in stratum oriens (s.o.) of $F m r 1^{-/ y}$ mice. Scale bar: $45 \mu \mathrm{m}$. (d) Immunodetection of p-p70S6K(T389) in $F m r 1^{-/ y}$ mice did not co-localize with GAD65/67 (GABAergic neurons, arrowheads). Scale bar: $50 \mu \mathrm{m}$. (e) p70S6K(T389) and Akt(S473) phosphorylation in hippocampal samples of WT and $F m r 1^{-/ y}$ mice ( $n=6$ per group). Data are expressed as mean \pm s.e.m. $* * P<0.01,{ }^{* * *} P<$ $0.001\left(\mathrm{Fmrl}^{-/ \mathrm{y}}\right.$ versus WT); \#P<0.05 (treatment versus vehicle). (f) Representative staining with DiOlistics of hippocampal dendrites in the CA1 field of the hippocampus and overall dendritic spine counts after pharmacological treatments. Scale bar: $2 \mu \mathrm{m}$. Data are expressed as mean \pm s.e.m. ${ }^{*} P<0.05\left(F m r l^{-/ y}\right.$ versus WT). (g) Morphological analysis of dendritic spines in the CA1 field of the hippocampus after pharmacological treatments. Data are expressed as mean \pm s.e.m. $* P<0.05, * * P<0.01, * * * P<0.001$ $\left(F m r l^{-/ y}\right.$ versus WT); \#P<0.05, \#\#\#P<0.001 (rimonabant versus vehicle).

\section{Figure 3. Prevention of other fragile $\mathrm{X}$ related phenotypes by pharmacological}

treatment. (a) Anxiety-like behavior in the elevated plus-maze test of WT and $\mathrm{Fmrl}^{-/ \mathrm{y}}$ mice ( $n=10-12$ per group) after receiving rimonabant. None of the treatments affected overall activity in the elevated plus-maze (Supplementary Fig. 6). (b) Anxiety-like behavior in the elevated plus-maze test of WT and $F m r l^{-/ y}$ mice $(n=10-12$ per group) after receiving MTEP. (c) Anxiety-like behavior in the elevated plus-maze test of WT and $F m r 1^{-/ y}$ mice ( $n=10-12$ per group) after receiving AM630. (d) Anxiety-like 
behavior in the elevated plus-maze test of WT and $F m r l^{-/ y}$ mice $(n=10-12$ per group) after receiving tesmirolimus. (e) Secondary nociceptive response to inflammatory pain of WT and $F m r 1^{-/ y}$ mice ( $n=12-14$ per group) after receiving rimonabant, AM630 and tesmirolimus. (f) Audiogenic seizure susceptibility of WT and $F m r l^{-/ y}$ mice $(n=6-8$ per group) after receiving rimonabant, AM630 and temsirolimus. Data are expressed as mean \pm s.e.m. $* P<0.05, * * P<0.01, * * * P<0.001\left(F m r 1^{-/ y}\right.$ versus $\left.\mathrm{WT}\right) ; \# P<0.05$, $\# \# P<0.01$ (treatment versus vehicle).

Figure 4. Genetic attenuation of CB1R corrects behavioral deficits and mTOR over-activation in $\mathrm{Fmrl}^{-/ \mathrm{y}}$ mice.

(a) Discrimination index values in the object-recognition test of $\mathrm{Fmrl}^{+/ \mathrm{y}} / \mathrm{Cnrl}^{+/+}$(WT) mice, $\mathrm{Fmrl}^{-/ \mathrm{y}} / \mathrm{Cnrl}^{+/+}\left(\mathrm{Fmrl}^{-/ \mathrm{y}}\right)$ and $\mathrm{Fmrl}^{-/ y} / \mathrm{Cnrl}^{+/-}$mice $(n=6-10$ per group). (b) Secondary nociceptive response to inflammatory pain in WT, $\mathrm{Fmrl}^{-\mathrm{y}}$, and $\mathrm{Fmrl}^{-}$ ${ }^{\mathrm{y}} / \mathrm{Cnrl}^{+/-}$mice ( $n=6-10$ per group). (c) Audiogenic seizure susceptibility of WT, $\mathrm{Fmrl}^{-/ \mathrm{y}}$, and $\mathrm{Fmrl}^{-/ \mathrm{y}} / \mathrm{Cnrl}^{+/-}$mice ( $n=6-10$ per group). (d) p-p70S6K(T389), p70S6K, CB1R, Fmrp and GAPDH detection in hippocampal samples of WT, Fmrl ${ }^{-/ y}$, and $\mathrm{Fmrl}^{-/ \mathrm{y}} / \mathrm{Cnrl}^{+/-}$mice $(n=6-10$ per group). Arrow indicates Fmrp detection, in contrast to the non-specific detection of an extra-band with lower molecular weight than Fmrp. Data are expressed as mean \pm s.e.m. ${ }^{*} P<0.05,{ }^{* *} P<0.01, * * * P<0.001\left(F m r 1^{-/ y}\right.$ versus WT); $\# P<0.05, \# \# P<0.01\left(F m r 1^{-/ y} / C n r l^{+/-}\right.$versus $\left.F m r l^{-/ y}\right)$. 


\section{Acknowledgements}

We thank C. Fernández-Avilés, D. Real, M. Linares and H. Gómez for expert technical assistance, and O.J. Manzoni for helpful comments. Fmrl KO mice in C57BL/6J background were kindly provided by D. Nelson at Baylor College of Medicine (Houston, Texas). AB-G is recipient of a predoctoral fellowship (Ministerio de Educación y Cultura). SM is recipient of a Ramón y Cajal contract (Ministerio de Educación y Cultura). This study was supported by grants from La Marató de TV3 (\#090910 to AO), Grants from the Ministerio de Ciencia e Innovación (\#SAF200907309 to AO, \#SAF2011-29864 to RM and SAF2010-21547 to CM); Instituto de Salud Carlos III (RD06/0001/0001 to RM); PLAN E (Plan Español para el Estímulo de la Economía y el Empleo); Generalitat de Catalunya (SGR-2009-00731 to RM and SGR2009-00718 to RdlT); ICREA (Institució Catalana de Recerca i Estudis Avançats) Academia to RM.

Conflict of interests: All authors report no biomedical financial interests or potential conflicts of interest. 


\section{Author contributions}

A.B-G. participated in experimental design, conducted biochemical and behavioral experiments and wrote the manuscript. M.G-G. conducted biochemical and behavioral experiments. T.G. performed dendritic spine morphology analysis. C.A-P. performed stereotaxic surgeries and M.D. provided Fmrl KO and WT mice and discussed the experiments. A.P. and R.dlT. measured endocannabinoid levels. S.M. designed and conducted electrophysiological experiments, and wrote the manuscript. A.P. and S.M. were in charge of electrophysiological equipment. C.M. funded the project. R.M. funded the project and wrote the manuscript. A.O. conceptualized, participated in experimental design, supervised, funded the project and wrote the manuscript. 


\section{a}

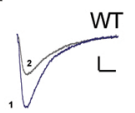

$\sqrt[2]{\frac{F m r 1^{-1}}{L}}$

, b

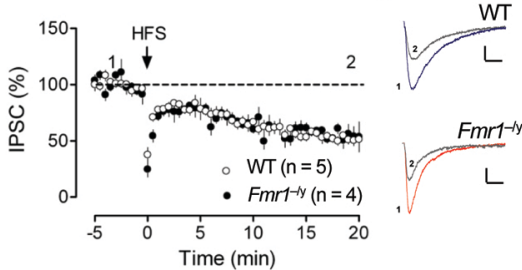

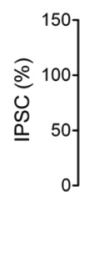

C

WT

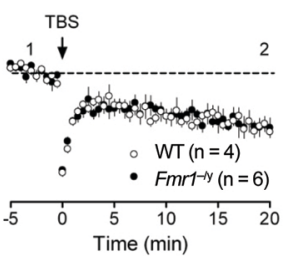

s.o.

Py

s.r.

Fmr1-y

d

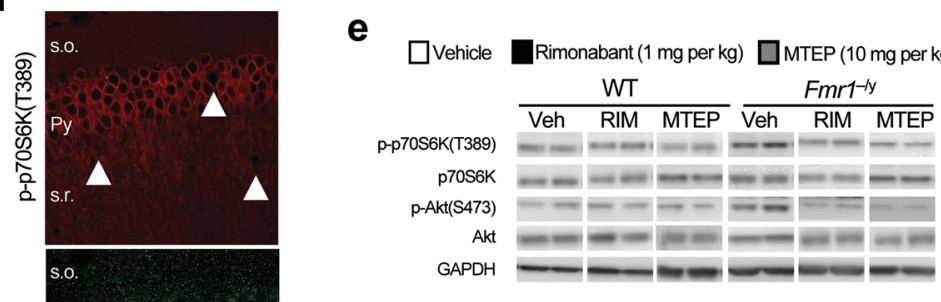

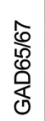

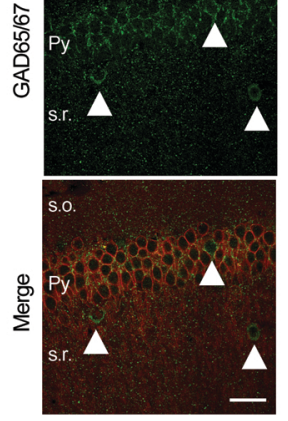

f
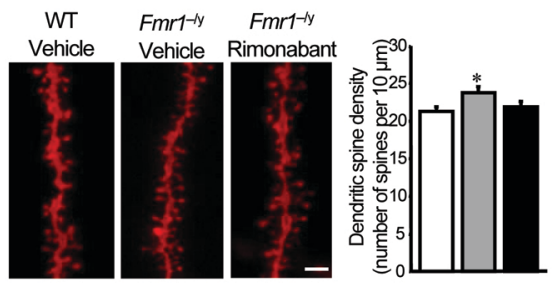

Figure 2. Busquets-Garcia_NMED_BC60101C

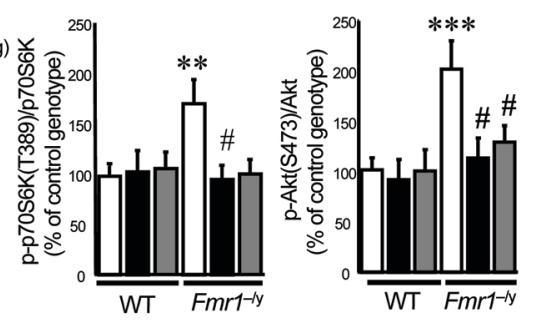

g

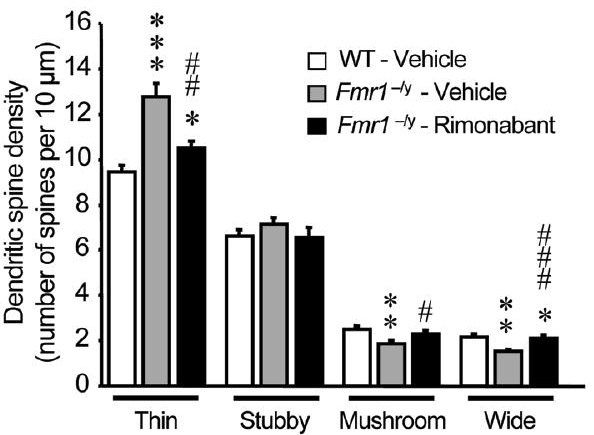



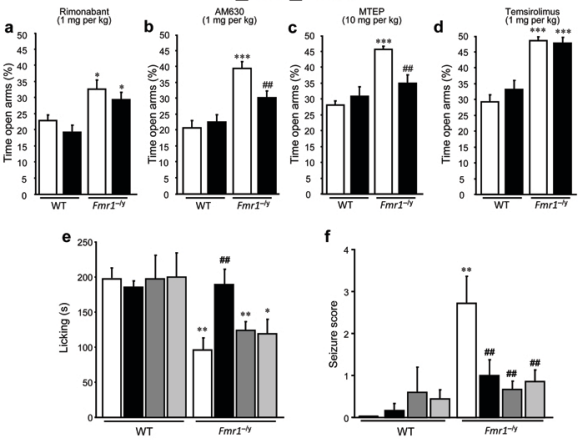

f

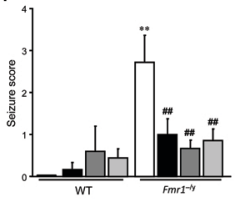

$\square$ vehicle $\quad$ Rimonabant (1 mg per $\mathrm{kg}$ )

$\square$ AMG630 (1 mg per kg) $\square$ Temsirolimus (1 mg per kg)

Figure 3. Busquets-Garcia_NMED_BC60101C 
a

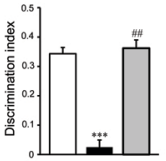

b

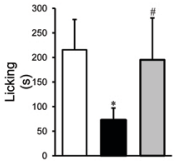

C

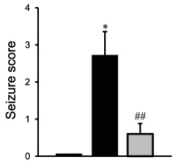

d
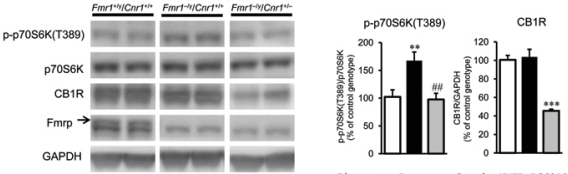

Figure 4. Busquets-Careia MMED BC60101C 